\title{
Prevalence of Mental Health Problems During Virus Epidemics in the General Public, Health Care Workers and Survivors: A Rapid Review of the Evidence
}

\author{
Simeon Joel Zürcher ${ }^{1,2 *}$, Philipp Kerksieck ${ }^{3}$, Christine Adamus ${ }^{1,2}$, \\ Christian Markus Burr ${ }^{1,2}$, Anja I. Lehmann ${ }^{3}$, Flavia Katharina Huber ${ }^{2}$ and Dirk Richter ${ }^{1,2,4}$ \\ ${ }^{1}$ Center for Psychiatric Rehabilitation, University Hospital for Mental Health (UPD), Bern, Switzerland, ${ }^{2}$ University Hospital of \\ Psychiatry and Psychotherapy, University of Bern, Bern, Switzerland, ${ }^{3}$ Public and Organizational Health, Epidemiology, \\ Biostatistics and Prevention Institute, University of Zurich, Zurich, Switzerland, ${ }^{4}$ Department of Health Professions, Bern \\ University of Applied Sciences, Bern, Switzerland
}

OPEN ACCESS

Edited by:

Tilman Steinert,

ZfP Südwürttemberg, Germany

Reviewed by:

Deborah Klein Walker,

Boston University, United States

Andreea Visla,

University of Zurich, Switzerland

Jakov Gather,

Ruhr University Bochum, Germany

*Correspondence:

Simeon Joel Zürcher simeon.zuercher@upd.unibe.ch

Specialty section:

This article was submitted to

Public Mental Health,

a section of the journal

Frontiers in Public Health

Received: 11 May 2020

Accepted: 09 October 2020

Published: 11 November 2020

Citation:

Zürcher SJ, Kerksieck P, Adamus C, Burr CM, Lehmann Al, Huber FK and Richter D (2020) Prevalence of Mental

Health Problems During Virus

Epidemics in the General Public, Health Care Workers and Survivors: A

Rapid Review of the Evidence.

Front. Public Health 8:560389.

doi: 10.3389/fpubh.2020.560389
Background: The swift spread of SARS-CoV-2 provides a challenge worldwide. As a consequence of restrictive public health measures like isolation, quarantine, and community containment, the provision of mental health services is a major challenge. Evidence from past virus epidemics and the current SARS-CoV-2 outbreak indicate high prevalence rates of mental health problems (MHP) as short- and long-term consequences. However, a broader picture of MHP among different populations is still lacking.

Methods: We conducted a rapid review on MHP prevalence rates published since 2000, during and after epidemics, including the general public, health care workers, and survivors. Any quantitative articles reporting on MHP rates were included. Out of 2,855 articles screened, a total of 74 were included in this review.

Results: Most original studies on MHP were conducted in China in the context of SARS-CoV-1, and reported on anxiety, depression, post-traumatic stress symptoms/disorder, general psychiatric morbidity, and psychological symptoms. The MHP rates across studies, populations, and epidemics vary substantially. While some studies show high and persistent rates of MHP in populations directly affected by isolation, quarantine, threat of infection, infection, or life-threatening symptoms (e.g., health care workers), other studies report minor effects. Furthermore, even less affected populations (e.g., distant to epidemic epicenter, no contact history with suspected or confirmed cases) can show high rates of MHP.

Discussion: MHP vary largely across countries and risk-groups in reviewed studies. The results call attention to potentially high MHP during epidemics. Individuals affected directly by an epidemic might be at a higher risk of short or even long-term mental health impairments. This study delivers insights stemming from a wide range of psychiatric instruments and questionnaires. The results call for the use of validated and standardized 
instruments, reference norms, and pre-post measurements to better understand the magnitude of the MHP during and after the epidemics. Nevertheless, emerging MHP should be considered during epidemics including the provision of access to mental health care to mitigate potential mental impairments.

Keywords: COVID-19, epidemic, mental health problems, pandemic, prevalence, SARS-CoV-2

\section{INTRODUCTION}

In the past two decades, many countries faced challenges in the realm of major infectious disease epidemics including SARS-CoV-1 (1), Swine flu (H1N1) (2), Middle East respiratory syndrome coronavirus (MERS-CoV) (3), avian influenza (H7N9) (4), Ebolavirus (5), and the recent worldwide SARS-CoV2 outbreak (6). Epidemic outbreaks can result in high case fatality rates and morbidity $(7,8)$ and may require communities to introduce restrictive public health measures like isolation, mass quarantine, and community containment interventions in order to stop transmissions and save lives (9). In consequence, epidemics can cause a high individual and societal burden and can lead to substantial economic loss (7, 10-12). While considerable efforts rely on protective and treatment measures such as virus transmission pathways, clinical presentations, and the development of vaccinations, attention is only recently given to short or long-term mental health problems (MHP, hereafter defined as psychiatric/psychological symptoms and mental illness/disorders) (13) that may arise due to the different surrounding consequences of an epidemic in the general public, health care workers (HCW), and survivors of infectious diseases (survivors).

Epidemics can negatively impact a substantial part of the general public in many different ways such as feelings of a personal threat of being infected $(7,14,15)$, worries about relatives and family members or losing loved ones (14-16), and protective measures like mass quarantining, the consequences of which leads to individual and social restrictions, and economic loss (14). As a result, these factors can elicit feelings of anxiety, anger, loneliness, grief, boredom and may lead to serious MHP (14, 15, 17). Furthermore, the extensive and sometimes controversial mass media coverage during epidemics may amplify uncertainty, loss of control and anxiety (14, 17). Aside from the general public, HCW are prone to different MHP since they usually face an immediate threat of infection through patient contact by working at the epidemic frontline. Studies suggest that HCW accounted for up to $57 \%$ of SARS-CoV-1, 27\% of MERS$\mathrm{CoV}$, and $12 \%$ of Ebola cases in some countries, which frequently resulted in morbidity or even death $(18,19)$. In HCW, epidemics often result in difficult working conditions like staff shortage, increased workload (7), overwhelming patient numbers $(7,19)$, limited safety equipment (7), and quarantine or isolation after infectious disease transmission $(7,14)$. Furthermore, HCW often suffer social consequences like stigma $(7,20,21)$, mistrust and violence (7) avoidance from relatives, and the fear of infecting others (21). Given the high risk of transmission, HCW often account for a substantial fraction of survivors, who frequently experience isolation, intensive treatment, stigmatization, and exposure to an immediate threat of morbidity or death (7, 22). To date, many studies exist that describe MHP related to epidemics across a wide range of populations. However, to the best of our knowledge no review covering MHP during epidemics currently exists.

\section{Objectives}

The purpose of this rapid review is to provide an overview of MHP prevalence rates during and after large epidemics of the past two decades. This research is important for informing research and practice about potential mental health issues and implications that may arise in the context of the current SARSCoV-2 epidemic. We aim to provide a broad picture of MHP that may arise across a wide range of populations including (a) the general public, (b) HCW, and (c) and virus disease survivors. To synthesizes and deliver context-sensitive knowledge, we used a rapid review approach. As compared to systematic reviews, rapid reviews are a form of systematic knowledge synthesis with accelerated review processes and streamlined methods aiming at providing relevant evidence in a timely and efficient manner (23).

\section{MATERIALS AND METHODS}

\section{Search Strategy}

The rapid and dynamic development of the current situation with SARS-CoV-2 requires quick evidence synthesis in order to inform decision-making processes in health care systems. The methodology of this article is based on the practical guide for rapid reviews provided by WHO. The results described in this study reflect a descriptive synthesis of evidence. As common for rapid reviews, facilitated methods for search, selection and data extraction were used and no meta-analysis was performed (23). We undertook a review of evidence on prevalence rates during and after epidemic outbreaks on MHP in the general public, HCW, and survivors. The focus was on SARS-CoV-1, H1N1, MERS-CoV, H7N9, Ebolavirus, and SARS-CoV-2. PubMed was searched on April 1, 2020 with a broad search strategy (see Supplementary Table 1). These virus epidemics were included as we assume important parallels in the way they affect mental health. More specifically, they elicit a large degree of uncertainty, feelings of threat, and major consequences in social and work lives.

\section{Participants, Interventions, and Comparators}

Any type of quantitative study that provided prevalence rates of MHP in adults ( $\geq 18$ years) during and after epidemic 
outbreaks, published in English from the year 2000 to March 31, 2020 was included. Studies that measured MHP rates assessed by psychometrically validated instruments, diagnostic interview, and medical records (chart review), were also included. We excluded studies that used a qualitative design, that did not report on MHP prevalence rates (e.g., providing mean scores only), that did not provide prevalence rates based on previously defined cut-off values for a measurement instrument (e.g., median based sample splitting), and that included MHP measured by single questions/items. Studies on common seasonal influenza were also excluded. Furthermore, general states like social functioning, quality of life, generic fears (e.g., fear of contracting a virus or worries) or stigma were excluded.

Based on the titles and abstracts of studies, potential eligible studies of the database search were selected by one author (CA) using a co-developed standardized review form to assess study eligibility. Subsequently, one author (SJZ) assessed full texts for eligibility. Doubts and uncertainty in eligibility of a certain study were solved by discussion (SJZ and CA).

\section{Data Sources, Study Selection, and Data Extraction}

An electronic data extraction form was developed to assess the characteristics of the included studies and the reported MHP prevalence rates. Data was extracted by four authors in parallel (SJZ, CA, PK, and FKH) and subsequently audited by another author. Collected items included: author(s), year of publication, country or region, number of participants, type of epidemic outbreak, time point of assessment, type of MHP assessed, MHP prevalence rate, and assessment method. Time point of assessment was coded as: during epidemic/hospital stay, postepidemic/discharge including one-year follow-up $(\leq 1 \mathrm{y})$, between 1 and 4 years follow-up (>1-4y), or a combination of both if applicable (e.g., for longitudinal studies). MHP were categorized into anxiety, depression, post-traumatic symptoms/disorders (PTSD) or stress, burnout, psychiatric morbidity, and further MHP like hallucinations or insomnia. We used baseline assessment data for intervention studies that provided prevalence rates. Data was stratified by the following populations: (a) general public including general surveys, (b) HCW including all hospital staff, military duty members, and family members as caregivers involved in active treatment or in potential contact with patients, and (c) infectious disease survivors (that may include suspected cases in some studies). Data quality and strength of evidence was not rated in the current review. All authors who extracted data discussed possible uncertainties with the primary reviewer SJZ.

\section{Data Analysis}

Included studies varied in assessment of MHP (e.g., questionnaires, diagnostic interviews), MHP instruments with applied cut-off scores, sampling methods and response rates, outbreak-related time points of assessments, and in regional differences in the magnitude/level of affect. Due to the approach chosen (rapid review), no meta-analysis was conducted. Therefore, a descriptive approach was utilized to synthesize reported MPH prevalence rates. If provided, we show
MHP rates from a moderate degree of severity as defined by authors within original studies.

\section{RESULTS}

\section{Study Characteristics}

Our PubMed search yielded 2,855 articles of which 2,630 articles were excluded based on title and abstract screening and 151 based on full-text screening. Most common reasons for exclusion during full-text screening were; (a) no prevalence rates provided (e.g., provision of mean scores for assessment instruments only), (b) mental health measured by single items (e.g., only one question used for assessment), (c) no specific mental health measures included (e.g., worries, concerns, quality of life), (d) qualitative design. Finally, 74 articles were included for the qualitative synthesis (see Figure 1). The majority of studies were cross-sectional in design and focused on MHP during SARSCoV-1 $(n=41)$, followed by Ebolavirus $(n=12)$, MERS-CoV and SARS-CoV-2 $(n=7), \mathrm{H} 1 \mathrm{~N} 1(n=6)$, and H7N9 $(\mathrm{n}=1)$. About half of the studies in the general public used random sampling, while the majority of articles in HCW and survivors were non-random samples. The vast majority of studies was conducted in China, including Taiwan and Hong Kong $(n=39)$, followed by other countries in Asia $(n=14)$, in Africa $(n=$ 12 ), and the American continent (mainly Canada; $n=6$ ), with three studies conducted in Europe. We found $n=28,26$, and 20 studies that investigated the general public, HCW, and survivors, respectively. The vast majority of studies assessed MHP using self-reported questionnaires, while only few used standardized diagnostic interviews. Results stratified by general public, HCW, and survivors can be found in Tables 1-3.

\section{Synthesized Findings General Public}

Range of prevalence rates across original articles were as follows: anxiety (0.7-47.2\%), depression (1.4-32.4\%), any anxiety/depression symptoms combined (48.6\%), PTSD/stress (2.0-76.4\%), and psychiatric morbidity $(8.0-26.2 \%)$. The rates of further MHP included any mental disorder $(<1.0 \%)$, alcohol/substance use disorders ( $<1.0 \%)$, anger (6.4-52.8\%), moderate to severe emotional disorder or depression $(12.0 \%)$, and psychotic symptoms like hallucinations (21.0\%). The highest and lowest rates of anxiety were found in MERS-CoV (48.6\%), and SARS-CoV-2 (0.7\%), respectively. For depression the highest rates were found in SARS-CoV-1 (32.4\%) and the lowest in Ebolavirus (1.4\%). For PTSD/stress, the highest rates were shown for Ebolavirus (76.4\%), and the lowest in H1N1 (2.0\%). Psychiatric morbidity was highest in SARS-CoV-1 (26.2\%) and lowest in $\mathrm{H} 1 \mathrm{~N} 1$ (8.0\%). The majority of studies in the general public reported on MHP during or shortly after $(\leq 1 \mathrm{y})$ epidemic outbreaks. To the best of our knowledge there are no studies published reporting on potential late sequela $>1 \mathrm{y}$ after an epidemic.

\section{Health Care Workers}

Range of prevalence rates were as follows: anxiety (1.5-88.0\%), depression (2.3-49.1\%), PTSD/stress (1.5-71.5\%), burnout 


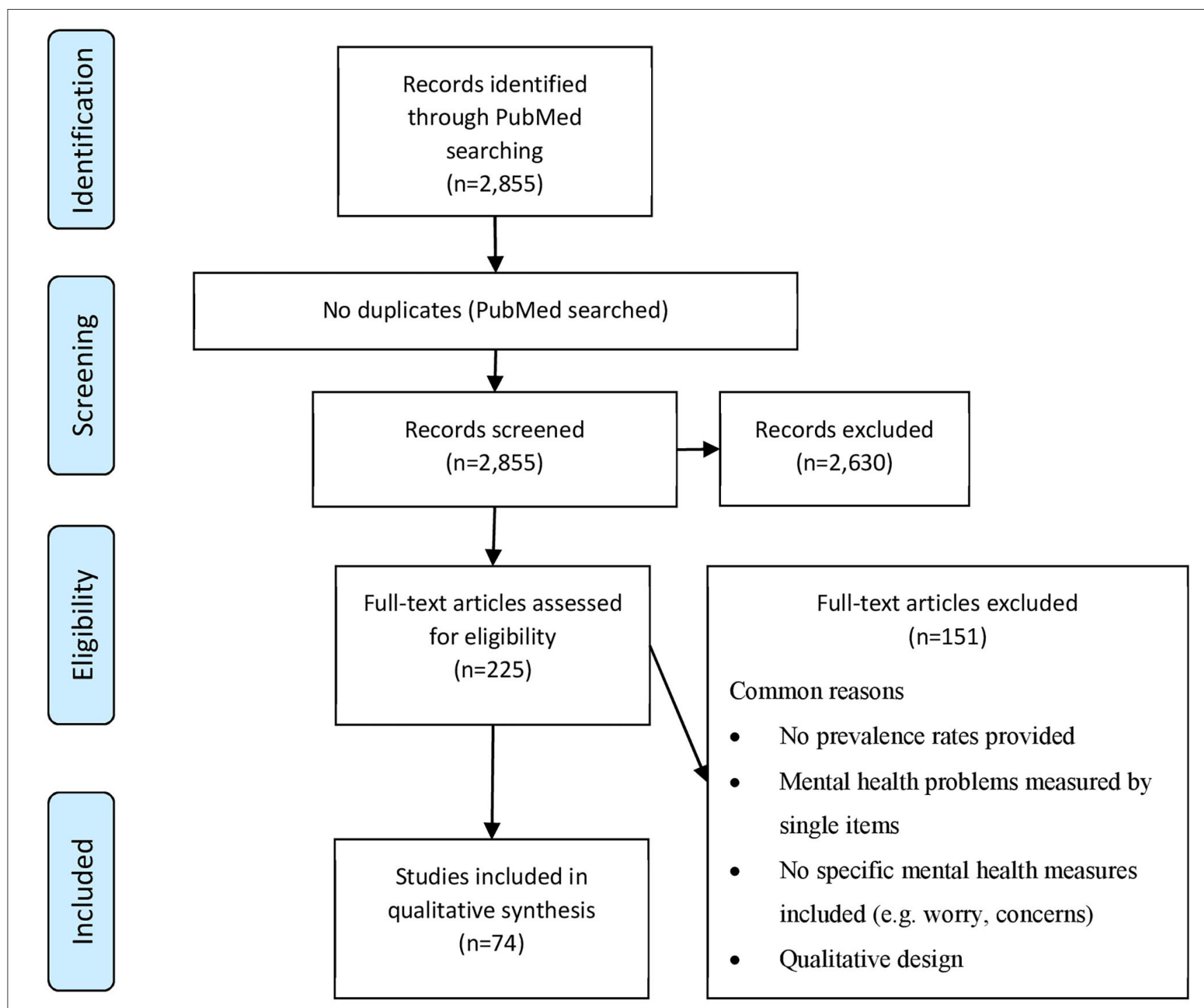

FIGURE 1 | PRISMA flow diagram of the selection process of studies reported on mental health problem prevalence rates during or after virus epidemics retrieved for the rapid review.

(19.2-30.4\%), and psychiatric morbidity (6.0-75.3\%). The rates of further $\mathrm{MPH}$ included any new Axis 1 diagnosis (6.8\%), insomnia (9.4-37.1\%), and substance abuse or alcohol related symptoms (1.5-19.0\%). The full range of rates in anxiety were both found in H1N1 (1.5-88.0\%). For depression, the highest rates were found in SARS-CoV-2 (49.1\%) and the lowest in Ebolavirus (2.3\%). For PTSD/stress, the highest rates were shown for SARS-CoV-2 (71.5\%) and the lowest for SARS-CoV-1 (1.5\%). Highest and lowest rates for psychiatric morbidity were both found for SARS-CoV-1 (6.0-75.3\%). The majority of studies in HCW reported on MHP during or shortly after $(\leq 1 \mathrm{y})$ epidemic outbreaks. Four studies reported on MHP with followup assessments of up to 4 years in the context of SARS-CoV-1. MHP differed substantially even when separated by follow-up time points. Results show that rates can still be high at follow-up time points $>1 y$ (e.g., Burnout rates of $30.4 \%$ ).

\section{Survivors}

Range of prevalence rates were as follows: anxiety (13.094.4\%), depression (11.0-50.5\%), PTSD/stress (1.2-96.2\%), and psychiatric morbidity (49.1-90.3\%). Furthermore, the rates of further MHP included any psychiatric diagnosis (33.3-58.9\%), fatigue (27.1-48.1\%), fear and panic (13.7$26.5 \%)$, hallucinations (1-5.9\%), insomnia (10.1-52.5\%), low mood (18.6-36.3\%). obsession-compulsion (15.6-83.3\%), panic disorder $(32.5 \%)$, paranoid ideation $(72.2 \%)$, somatoform pain disorder (36.4\%), suicidal ideation (2.0\%), and tensions/hostility (20.6-94.4\%). The highest and lowest rates of anxiety were fund in Ebolavirus (94.4\%), and SARS-CoV-1/Ebolavirus (13\%), respectively. Depression was highest in SARS-CoV-1 (50.5\%) and lowest in ebolavirus (11\%). For PTSD/stress, the highest rates were shown for SARS-CoV-2 (96.2\%) and lowest for Ebolavirus (1.2\%). Psychiatric morbidity was described only in SARS-CoV-1 
TABLE 1 | Reported prevalence rates with severity of mental health problems in the general public during and after epidemic outbreaks since 2000 in the respective countries/regions.

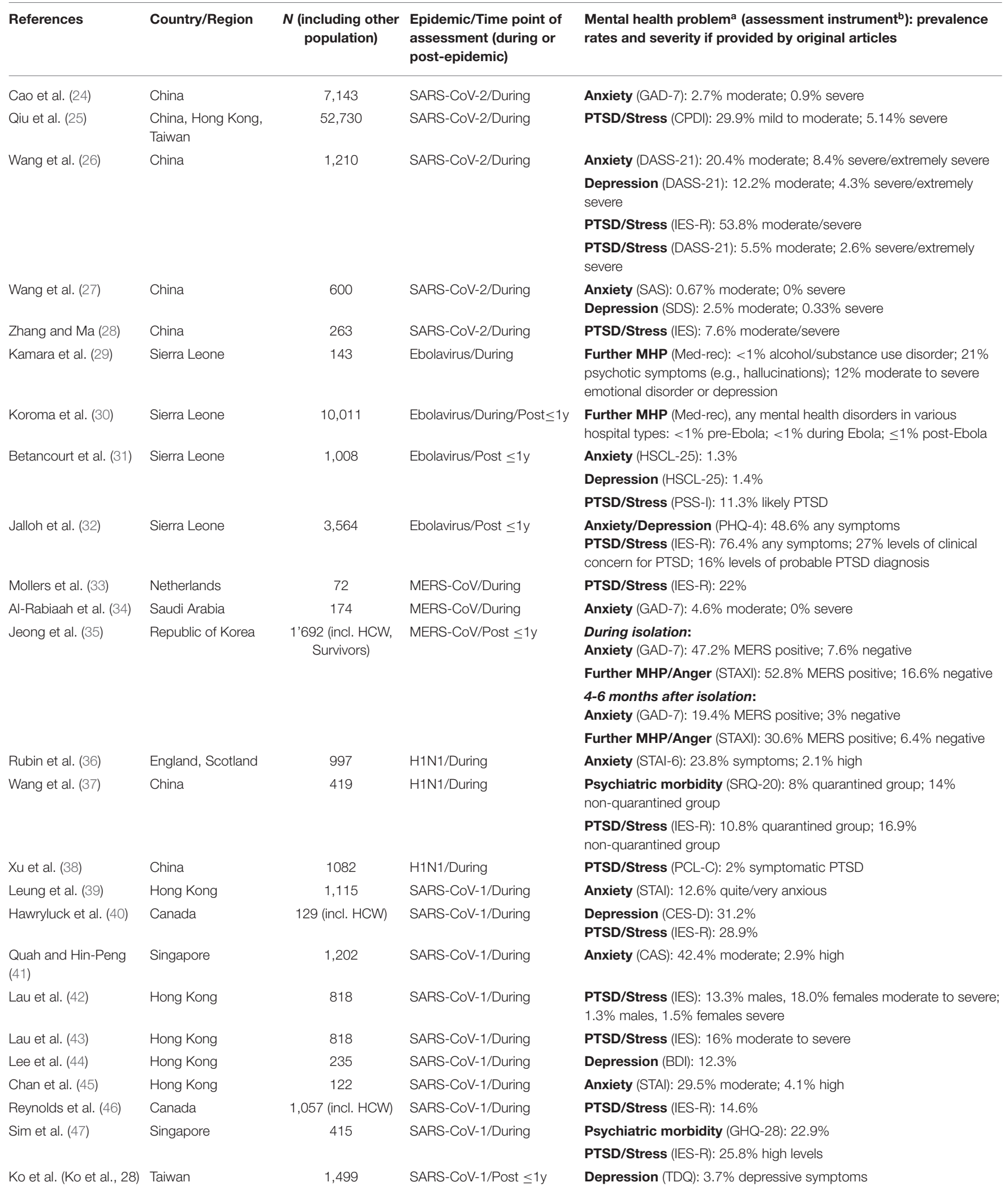


TABLE 1 | Continued

\begin{tabular}{|c|c|c|c|c|}
\hline References & Country/Region & $\begin{array}{l}N \text { (including other } \\
\text { population) }\end{array}$ & $\begin{array}{l}\text { Epidemic/Time point of } \\
\text { assessment (during or } \\
\text { post-epidemic) }\end{array}$ & $\begin{array}{l}\text { Mental health problem }{ }^{a} \text { (assessment instrument }{ }^{b} \text { ): prevalence } \\
\text { rates and severity if provided by original articles }\end{array}$ \\
\hline Lee et al. (48) & Hong Kong & 146 & SARS-CoV-1/Post $\leq 1 y$ & $\begin{array}{l}\text { Depression (CES-D): } 32.4 \% \text { elderly; } 18.7 \% \text { middle-aged } \\
\text { PTSD/Stress (IES-R): } 14.1 \% \text { elderly; } 4 \% \text { middle-aged }\end{array}$ \\
\hline Mihashi et al. (49) & China & 187 & SARS-CoV-1/Post $\leq 1 \mathrm{y}$ & $\begin{array}{l}\text { Psychiatric morbidity (GHQ-30): } 24.6 \% \text { during the isolation period; } \\
26.2 \% \text { during the recovery period }\end{array}$ \\
\hline Peng et al. (50) & Taiwan & 1,278 & SARS-CoV-1/Post $\leq 1 \mathrm{y}$ & PTSD/Stress (BSRS-5): 11.7\% \\
\hline
\end{tabular}

aPTSD, Post-traumatic stress disorder.

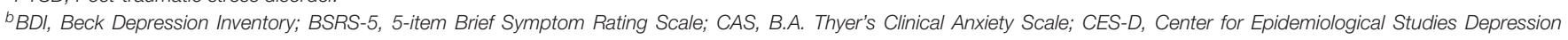

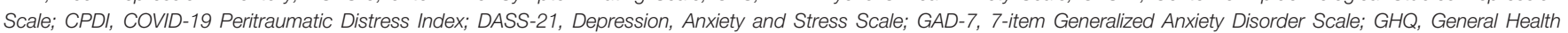

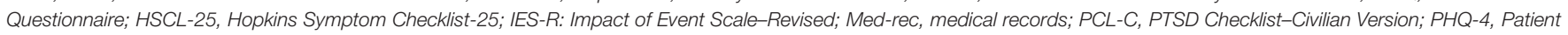

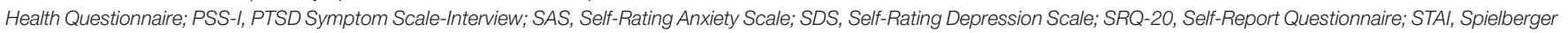
State-Trait Anxiety Inventory; STAXI, State-Trait Anger Expression Inventory; TDQ, Taiwanese Depression Questionnaire.

(49.1-90.3\%). As in HCW, studies in survivors across different follow-up time points show a broad range of MHP rates. Studies including assessments $>1 \mathrm{y}$ post-epidemic show that rates can still be high.

\section{DISCUSSION}

\section{Summary of Main Findings}

This rapid review presents a descriptive synthesis of 74 original articles using streamlined review methodology in order to provide a broad overview of MHP in a timely manner. We found a wide range of MHP including anxiety, depression, PTSD and stress related symptoms or disorders, psychiatric morbidity, and many further MHP like paranoid ideation, hallucinations, and insomnia that may occur in the general public, HCW or survivors during and after epidemic outbreaks. Original articles commonly describe simple prevalence rates rather than reporting changes in MHP since epidemic outbreaks. Aside from methodological issues and the large heterogeneity of original studies (e.g., poor validation, different cut-offs for case definition), which makes it difficult to understand the magnitude of the problem, MHP can be more prevalent in all three populations in the context of an epidemic. These problems may be substantial and can persist over time in HCW and survivors more directly affected by the epidemic threat. However, it should be noted that epidemic circumstances can also yield positive impacts on mental health like spending more time on physical activity and taking more care of one's mental health (43).

\section{General Public}

MHP ranged widely both across the general public and in all epidemics, which makes it difficult to estimate the magnitude and associated characteristics that may aggravate MHP. However, many studies investigated risk and protective factors of MHP. Although some controversy exists among studies, a higher level of epidemic exposure (e.g., living proximity to epidemic epicenter, contact history to high prevalent virus regions) (48, 94), hospitalization during epidemic (47), being quarantined (95), or having infected family members $(24,38,44)$ may aggravate MHP. Further risk factors include being female
(37, 38, 90, 94), chronic physical illness (85), poor self-rated health (26), and dissatisfaction with measures controlling the virus (37). Furthermore, many studies reported problems like loneliness, boredom, anger, worries about family members (26), and financial problems or economic loss $(3,42,56,96)$ that negatively interfere with mental health. In contrast, accurate health information (e.g., treatment, local outbreak situation) (26), particular precautionary measures (e.g., hand hygiene, wearing a mask) (26), social support $(24,43,95)$, and appraisals and coping strategies $(15,85)$ may be protective.

\section{Health Care Workers and Survivors}

Similarly, HCW and survivors showed a wide range of mental health impacts. However, MHP rates in these populations may be more substantial than in the general public. HCW that were directly involved in patient care (21), working in high risk units and with infected patients $(62,64,71,97)$ conscripted workers (62), or that underwent quarantine during outbreak $(20,74)$ were found to be associated with a higher risk of MHP. Furthermore, younger age $(21,64)$, being single $(59,74)$, fear of adversely affecting relatives $(97,98)$, pre-exposure to traumatic events or history of MHP $(64,70,74)$ were also found to be associated with a higher risk of MHP. In contrast, adequate professional education and training $(53,70,71)$, support from colleagues (59), appropriate information and communication (directives, precautionary measures, disease information) (59), and altruistic risk acceptance (74) were found to be protective. In survivors MHP may be aggravated by a history of mental illness (35), the fear of permanent damage or death $(85,88)$, longer duration of quarantine (40), having physical late sequelae (81), and impairment of ability to work (92). Furthermore, survivors that are HCW were shown to be more susceptible to long term MHP compared to non-HCW survivors $(90,99)$.

\section{Mental Health Problems and Methodological Issues}

The methodological characteristics and quality of studies in assessing MHP ranges widely. We found only few studies that did not utilize a cross-sectional design without repetition. Further, most cross-sectional studies did not report any comparative data 
TABLE 2 | Reported prevalence rates with severity of mental health problems in health care workers during and after epidemic outbreaks since 2000 in the respective countries/regions.

\begin{tabular}{|c|c|c|c|c|}
\hline References & Country/Region & $\begin{array}{l}N \text { (including other } \\
\text { population) }\end{array}$ & $\begin{array}{l}\text { Epidemic/Time point of } \\
\text { assessment (during or } \\
\text { post-epidemic) }\end{array}$ & $\begin{array}{l}\text { Mental health problem }{ }^{\mathrm{a}} \text { (assessment instrument } \mathrm{t}^{\mathrm{b}} \text { ): prevalence } \\
\text { rates and severity if provided by original articles }\end{array}$ \\
\hline Lai et al. (51) & China & 1,257 & SARS-CoV-2/During & $\begin{array}{l}\text { Anxiety (GAD-7): 32.3\% mild; 7\% moderate; 5.3\% severe } \\
\text { Depression (PHQ-9): } 35.6 \% \text { mild; } 8.6 \% \text { moderate; } 4.9 \% \text { severe } \\
\text { PTSD/Stress (IES-R): } 36.5 \% \text { mild; } 24.5 \% \text { moderate; } 10.5 \% \text { severe } \\
\text { Further MHP/Insomnia (ISI): } 26.2 \% \text { mild; } 6.8 \% \text { moderate; } 1 \% \text { severe }\end{array}$ \\
\hline Sipos et al. (52) & Liberia & 173 & Ebolavirus/During & $\begin{array}{l}\text { Anxiety (GAD-7): } 2.3 \% \\
\text { Depression (PHQ-8): } 2.3 \% \\
\text { PTSD/Stress (PCL): } 4.0 \% \\
\text { Further MHP/Insomnia (ISI): } 12.4 \%\end{array}$ \\
\hline Tang et al. (53) & China & 102 & H7N9/During & PTSD/Stress (PCL-C): 20.6\% \\
\hline Lee et al. (54) & Repuplic of Korea & $\begin{array}{l}359 \text { during; } \\
77 \text { after }\end{array}$ & MERS-CoV/During & $\begin{array}{l}\text { During hospital shutdown: } \\
\text { PTSD/Stress (IES-R): } 64.1 \% \text { symptoms of; } 51.5 \% \text { diagnosis of PTSD } \\
1 \text { month after hospital shutdown (in those with PTSD diagnosis): } \\
\text { Anxiety (HADS): } 11 \% \\
\text { Depression (HADS): } 15.1 \% \\
\text { PTSD/Stress (IES-R): } 54.5 \% \text { symptoms of; } 40.3 \% \text { diagnosis of PTSD } \\
\text { Psychiatric morbidity (MINI): } 5.5 \% \text { major depression; } 11 \% \\
\text { generalized anxiety disorder }\end{array}$ \\
\hline Jung et al. (55) & Repuplic of Korea & 147 & MERS-CoV/Post $\leq 1 \mathrm{y}$ & PTSD/Stress (IES-R): $57.1 \%$ total; $32.0 \%$ moderate; $25.1 \%$ full PTSD \\
\hline Mishra et al. (56) & India & 271 & H1N1/During & Anxiety (BAl): 1.5\% moderate/high \\
\hline $\begin{array}{l}\text { Elizarraras-Rivas et al. } \\
\text { (57) }\end{array}$ & Mexico & 35 & H1N1/Post $\leq 1 y$ & $\begin{array}{l}\text { Anxiety (DAQ): } 71 \% \text { moderate; } 17 \% \text { high } \\
\text { Depression (CES-D): } 34 \% \text { low; } 6 \% \text { moderate; } 3 \% \text { high } \\
\text { PTSD/Stress (PSS-10): 0\% moderate; } 3 \% \text { high }\end{array}$ \\
\hline Goulia et al. (58) & Greece & 469 & H1N1/Post $\leq 1 y$ & $\begin{array}{l}\text { Psychiatric morbidity/stress (GHQ-28): } 20.7 \% \text { mild/moderate; } 6.8 \% \\
\text { severe }\end{array}$ \\
\hline Bai et al. (20) & Taiwan & 338 & SARS-CoV-1/During & PTSD/Stress (DSM-IV): $5 \%$ acute stress disorder \\
\hline Chan and Huak (59) & Singapore & 661 & SARS-CoV-1/During & $\begin{array}{l}\text { Psychiatric morbidity (GHQ-28): } 27 \% \\
\text { PTSD/Stress (IES): } 20 \%\end{array}$ \\
\hline Chong et al. (60) & Taiwan & 1257 & SARS-CoV-1/During & Psychiatric morbidity (GHQ-12): 75.3\% \\
\hline Nickell et al. (61) & Canada & 2,001 & SARS-CoV-1/During & Psychiatric morbidity (GHQ-12): 29\% \\
\hline Verma et al. (21). & Singapore & 1,050 & SARS-CoV-1/During & $\begin{array}{l}\text { Psychiatric morbidity (GHQ-28): 14.1\% of general practitioners; } 6 \% \\
\text { TCM practitioners }\end{array}$ \\
\hline Chen et al. (62) & Taiwan & 131 & SARS-CoV-1/During & $\begin{array}{l}\text { PTSD/Stress (IES): 11\% total; 17\% in high-risk units; 10\% in low risk } \\
\text { units }\end{array}$ \\
\hline Lu et al. (63) & Taiwan & 127 & SARS-CoV-1/During & Psychiatric morbidity (GHQ-12): $17.3 \%$ \\
\hline Su et al. (64) & Taiwan & 102 & SARS-CoV-1/During & $\begin{array}{l}\text { Depression (BDI): } 27.5 \% \text { total; } 38.5 \% \text { in SARS units; } 6.7 \% \text { in } \\
\text { non-SARS units } \\
\text { PTSD/Stress (DTS-C): } 33 \% \text { SARS units; } 19 \% \text { non-SARS units } \\
\text { Further MHP/Insomnia (PSQI): } 37.1 \% \text { SARS units; } 9.4 \% \text { non-SARS } \\
\text { units }\end{array}$ \\
\hline Tam et al. (65) & Hong Kong & 652 & $\begin{array}{l}\text { SARS-CoV-1/During/Post } \\
\leq 1 y\end{array}$ & Psychiatric morbidity (GHQ-12): 56.7\% \\
\hline Lung et al. (66) & Taiwan & $\begin{array}{l}\text { During } 127 \\
\text { Follow-up } 123\end{array}$ & $\begin{array}{l}\text { SARS-CoV-1/During/Post } \\
\leq 1 y\end{array}$ & $\begin{array}{l}\text { During epidemic: } \\
\text { Psychiatric morbidity (CHQ-12): } 17.3 \% \\
\text { 1-year follow-up: } \\
\text { Psychiatric morbidity (CHQ-12): } 15.4 \%\end{array}$ \\
\hline Sim et al. (67). & Singapore & 277 & SARS-CoV-1/Post $\leq 1 y$ & $\begin{array}{l}\text { Psychiatric morbidity (GHQ-28): } 20.6 \% \\
\text { PTSD/Stress (IES-R): } 9.4 \%\end{array}$ \\
\hline Phua et al. (68) & Singapore & 96 & SARS-CoV-1/Post $\leq 1 y$ & $\begin{array}{l}\text { Psychiatric morbidity (GHQ-28): } 18.8 \% \\
\text { PTSD/Stress (IES): } 17.7 \%\end{array}$ \\
\hline
\end{tabular}


TABLE 2 | Continued

\begin{tabular}{|c|c|c|c|c|}
\hline References & Country/Region & $\begin{array}{l}N \text { (including other } \\
\text { population) }\end{array}$ & $\begin{array}{l}\text { Epidemic/Time point of } \\
\text { assessment (during or } \\
\text { post-epidemic) }\end{array}$ & $\begin{array}{l}\text { Mental health problem }{ }^{a} \text { (assessment instrument }{ }^{b} \text { ): prevalence } \\
\text { rates and severity if provided by original articles }\end{array}$ \\
\hline \multirow[t]{2}{*}{ Lin et al. (69) } & Taiwan & 92 & SARS-CoV-1/Post $\leq 1 \mathrm{y}$ & PTSD/Stress (DTS-C): 19.3\% likely PTSD \\
\hline & & & & Psychiatric morbidity (GHQ-12): 47.8\% \\
\hline \multirow[t]{3}{*}{ Lancee et al. (70) } & Canada & 133 & SARS-CoV-1/Post $\leq 1 y$ & Depression (SCID): 3.8\% major depression \\
\hline & & & & Stress/PTSD (SCID): 1.5\% \\
\hline & & & & $\begin{array}{l}\text { Further MHP (SCID): } 0.8 \% \text { panic disorder; } 1.5 \% \text { substance } \\
\text { abuse/dependence; } 6.8 \% \text { any new axis I diagnosis }\end{array}$ \\
\hline \multirow[t]{3}{*}{ Maunder et al. (71) } & Canada & $\begin{array}{l}587 \text { exposed; } \\
182 \text { non exposed }\end{array}$ & SARS-CoV-1/Post >1-4y & $\begin{array}{l}\text { PTSD/Stress (IES): } 13.8 \% \text { high in exposed; } 8.4 \% \text { high in } \\
\text { non-exposed group }\end{array}$ \\
\hline & & & & $\begin{array}{l}\text { PTSD/Stress (K10): } 44.9 \% \text { high in exposed; } 30.2 \% \text { high in } \\
\text { non-exposed }\end{array}$ \\
\hline & & & & $\begin{array}{l}\text { Burnout (MBI-EE): } 30.4 \% \text { high in exposed; 19.2\% high in } \\
\text { non-exposed }\end{array}$ \\
\hline \multirow[t]{3}{*}{ Wu et al. (72) } & China & 549 & SARS-CoV-1/Post >1-4y & Depression (CES-D): 22.8\% \\
\hline & & & & PTSD/Stress (IES-R): 10.1\% high PTSD symptoms \\
\hline & & & & Further MHP/Alcohol-related symptoms (NHSDA-adapted): 19\% \\
\hline Wu et al. (73) & China & 549 & SARS-CoV-1/Post >1-4y & $\begin{array}{l}\text { PTSD/Stress (IES-R): } 10 \% \text { high level at any time during follow-up } \\
\text { period; } 4 \% \text { still had high level at 3-year follow-up }\end{array}$ \\
\hline \multirow[t]{2}{*}{ Liu et al. (74) } & China & 549 & SARS-CoV-1/Post >1-4y & Depression (CES-D): 14\% moderate; 8.8\% high level \\
\hline & & & & PTSD/Stress (IES-R): 10\% high level \\
\hline
\end{tabular}

aPTSD, Post-traumatic stress disorder.

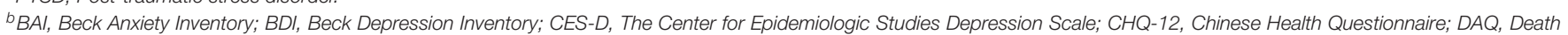

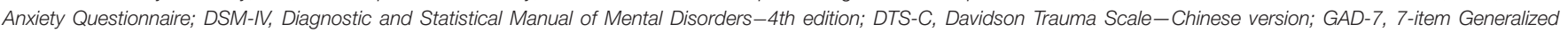

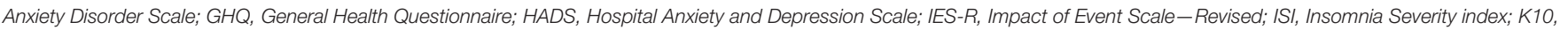

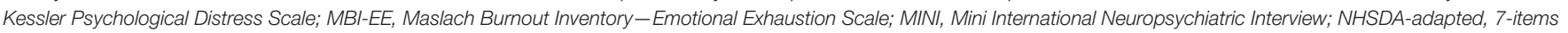

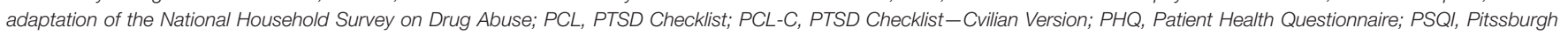
Sleep Quality Index; PSS-10, Perceived Stress Scale; SCID, Structured Clinical Interview for DSM-IV.

from which the change of prevalence rates due to the epidemic could be estimated. Sampling characteristics were also varying. Only about half of the studies in the general public were based on representative samples. As many studies were conducted during or shortly after the peak phase of the epidemic, results have to be regarded as acute stress reactions that do not allow for inference of longer-lasting MHP. While some authors used well-established and widely used instruments and standardized diagnostic interviews [e.g., Ji et al. (76) or Lancee et al. (70)], others used instruments with unclear quality [e.g., Guetiya Wadoum et al. (79)]. Besides the possibility of biased results, this approach makes it challenging to identify clinically relevant cases. With respect to the application of diagnostic instruments, cutoff values might vary between countries and cultures. Therefore, a lack of validated, country-specific, cut-off values of the measurement instruments might be problematic (32).

\section{Future Directives and Implications for Research, Policy, and Practice Monitoring MHP as a Tool for Mental Health Care Provision}

As shown by this review, MHP may be prevalent across a broad range of populations. In this vein, clinical monitoring of risk groups that are vulnerable to psychological impairments due to the current SARS-CoV-2 epidemic is essential (100).
Pfefferbaum and North (100) pointed out, that the monitoring of psychosocial needs should assess SARS-CoV-2-related stressors, secondary adversities, psychosocial effects, and indicators of vulnerability. Besides others, routine outcome monitoring (101) as a measurement feedback system, apps for (self-)monitoring of mood, sleep-quality, or medication adherence (102), and artificial intelligence predicting relevant psychiatric outcomes (103), are available for public mental health monitoring. In the best case, mental health service providers should be aided by e-monitoring during epidemics. As mentioned above, in research MHP should be assessed by standardized diagnostic interviews or measurement instruments, enabling appropriate case detection identifying risk groups in order to inform policy and practice. For profound and substantial planning of the mental health infrastructure, MHP associated with SARS-CoV2 need to be identified regarding potential evolving short or long term treatments.

\section{Access to Mental Health Service in Epidemics}

Furthermore, access to mental health services for those in need is paramount during the SARS-CoV-2 crisis, especially when social isolation is experienced (104). Beside the psychosocial consequences of public health measures such as quarantine (14), acute viral infection is unknown but likely to be accompanied by substantial neuropsychiatric symptoms (anxiety, depression, 
TABLE 3 | Reported prevalence rates with severity of mental health problems in survivors during and after epidemic outbreaks since 2000 in the respective countries/regions.

\begin{tabular}{|c|c|c|c|c|}
\hline References & Country/Region & $\begin{array}{l}N \text { (including other } \\
\text { population) }\end{array}$ & $\begin{array}{l}\text { Epidemic/Time point of } \\
\text { assessment (during or } \\
\text { post-epidemic) }\end{array}$ & $\begin{array}{l}\text { Mental health problem }{ }^{a} \text { (Assessment Instrument }{ }^{b} \text { ): Prevalence } \\
\text { rates and severity if provided by original articles }\end{array}$ \\
\hline Bo et al. (75) & China & 714 & SARS-CoV-2/During & PTSD/Stress (PCL-C): 96.2\% significant symptoms \\
\hline \multirow[t]{2}{*}{ Ji et al. (76) } & Sierra Leone & 18 & Ebolavirus/During & Anxiety (SCL-90-R): $94.4 \%$ phobic anxiety; $83.3 \%$ anxiety \\
\hline & & & & $\begin{array}{l}\text { Further MHP (SCL-90-R): } 83.3 \% \text { obsession-compulsion; } 94.4 \% \\
\text { hostility; } 72.2 \% \text { paranoid ideation }\end{array}$ \\
\hline \multirow[t]{3}{*}{ Howlett et al. (77) } & Sierra Leone & 35 & Ebolavirus/During & Anxiety (MINI-plus/MMSE): $27.5 \%$ anxiety symptoms \\
\hline & & & & Depression (MINI-plus/MMSE): 30\% depressive symptoms \\
\hline & & & & Further MHP/Insomnia (MINI-plus/MMSE): 52.5\% \\
\hline Etard et al. (78) & Guinea & 713 & Ebolavirus/Post $\leq 1 \mathrm{y}$ & Depression (CES-D): 17\% \\
\hline $\begin{array}{l}\text { Guetiya Wadoum et al. } \\
\text { (79) }\end{array}$ & Sierra Leone & 246 & Ebolavirus/Post $\leq 1 y$ & $\begin{array}{l}\text { Further MHP (ESMHCMAF): } 3.3 \% \text { hallucinations; } 24.4 \% \\
\text { psychotrauma; } 10.1 \% \text { insomnia }\end{array}$ \\
\hline \multirow[t]{2}{*}{ Keita et al. (80) } & Guinea & 256 & Ebolavirus/Post $\leq 1 \mathrm{y}$ & Depression (CES-D/ICD-10): 15\%; 10.9\% \\
\hline & & & & PTSD (ICD-10): $1.2 \%$ \\
\hline Pers et al. (81) & Guinea & 142 & Ebolavirus/Post >1-4y & Depression (CES-D): 18.3\% \\
\hline \multirow{3}{*}{$\begin{array}{l}\text { de St Maurice et al. } \\
\text { (82) }\end{array}$} & Liberia & 329 & Ebolavirus/Post $\leq 1-4 y$ & Anxiety (Med-rec): 13\% \\
\hline & & & & Depression (Med-rec): 13\% \\
\hline & & & & Insomnia (Med-rec): 15\% \\
\hline Kim et al. (83) & Republic of Korea & 27 & MERS-CoV/During & Depression (PHQ-9): 40.7\% \\
\hline \multirow[t]{7}{*}{ Lee et al. (84) } & Republic of Korea & 72 & MERS-CoV/Post $>1-4 y$ & $\begin{array}{l}12 \text { months follow-up: } \\
\text { Depression (PHQ-9): } 26.9 \%\end{array}$ \\
\hline & & & & PTSD/Stress (IES-R): 42.3\% \\
\hline & & & & Further MHP/Fatigue (FSS): $48.1 \%$ \\
\hline & & & & 18 months follow-up: \\
\hline & & & & Depression (PHQ-9): 17.3\% \\
\hline & & & & PTSD/Stress (IES-R): 26.9\% \\
\hline & & & & Further MHP/Fatigue (FSS): $32.7 \%$ \\
\hline \multirow[t]{2}{*}{ Cheng et al. (85) } & Hong Kong & 180 (incl. HCW) & SARS-CoV-1/Post $\leq 1 y$ & $\begin{array}{l}\text { Anxiety (BAl): } 23.4 \% \text { mild/moderate; } 24.6 \% \text { moderate/severe; } 7.3 \% \\
\text { severe }\end{array}$ \\
\hline & & & & $\begin{array}{l}\text { Depression (BDI): } 24.7 \% \text { mild/moderate; } 19.1 \% \text { moderate/severe; } \\
6.7 \% \text { severe }\end{array}$ \\
\hline \multirow[t]{4}{*}{ Sheng et al. (86) } & Hong Kong & 102 (incl. HCW) & SARS-CoV-1/Post $\leq 1 y$ & $\begin{array}{l}\text { Acute phase: } \\
\text { Further MHP (NPSC, examples): } 46.1 \% \text { insomnia; } 36.3 \% \text { low mood; } \\
2 \% \text { suicidal idea; } 26.5 \% \text { fear and panic; } 36.3 \% \text { tension; } 5.9 \% \\
\text { hallucinations }\end{array}$ \\
\hline & & & & Convalescent phase: \\
\hline & & & & Psychiatric morbidity (GHQ-28): 64.7\% \\
\hline & & & & $\begin{array}{l}\text { Further MHP (NPSC, examples): } 22.5 \% \text { insomnia; } 18.6 \% \text { low mood; } \\
\text { 0\% suicidal idea; } 13.7 \% \text { fear and panic; } 20.6 \% \text { tension; } 1 \% \\
\text { hallucinations }\end{array}$ \\
\hline \multirow[t]{7}{*}{ Wu et al. (87) } & Hong Kong & 131 (incl. HCW) & SARS-CoV-1/Post $\leq 1 y$ & $\begin{array}{l}1 \text { month follow-up: } \\
\text { Anxiety (HADS): } 13 \%\end{array}$ \\
\hline & & & & Depression (HADS): 18\% \\
\hline & & & & $\begin{array}{l}\text { PTSD/Stress (IES-R): } 4 \% \text { all subscales; } 12 \% \text { intrusion; } 9 \% \text { avoidance; } \\
15 \% \text { hyperarousal }\end{array}$ \\
\hline & & & & 3 months follow-up: \\
\hline & & & & Anxiety (HADS): 14\% \\
\hline & & & & Depression (HADS): 13\% \\
\hline & & & & $\begin{array}{l}\text { PTSD/Stress (IES-R): } 5 \% \text { all subscales; } 10 \% \text { intrusion; 8\% avoidance; } \\
9 \% \text { hyperarousal }\end{array}$ \\
\hline \multirow[t]{3}{*}{ Wu et al. (88) } & Hong Kong & 195 & SARS-CoV-1/Post $\leq 1 \mathrm{y}$ & Anxiety (HADS): 14\% \\
\hline & & & & Depression (HADS): 18\% \\
\hline & & & & PTSD/Stress (IES-R): 6\% \\
\hline
\end{tabular}


TABLE 3 | Continued

\begin{tabular}{|c|c|c|c|c|}
\hline References & Country/Region & $\begin{array}{l}N \text { (including other } \\
\text { population) }\end{array}$ & $\begin{array}{l}\text { Epidemic/Time point of } \\
\text { assessment (during or } \\
\text { post-epidemic) }\end{array}$ & $\begin{array}{l}\text { Mental health problem }{ }^{a}\left(\text { Assessment Instrument }{ }^{b}\right) \text { : Prevalence } \\
\text { rates and severity if provided by original articles }\end{array}$ \\
\hline \multirow[t]{3}{*}{ Kwek et al. (89) } & Singapore & 63 (incl. HCW) & SARS-CoV-1/Post $\leq 1 y$ & Anxiety (HADS): $17.5 \%$ at least moderate anxiety \\
\hline & & & & Depression (HADS): $11.1 \%$ at least moderate depression \\
\hline & & & & PTSD/Stress (IES): $41.7 \%$ at least moderate; $36.7 \%$ at least severe \\
\hline \multirow[t]{4}{*}{ Lee et al. (90) } & Hong Kong & 96 (incl. HCW) & SARS-CoV-1/Post $\leq 1 y$ & Anxiety (DASS-21): $36.7 \%$ moderate/severe; $14.4 \%$ extremely severe \\
\hline & & & & $\begin{array}{l}\text { Depression (DASS-21): 36.3\% moderate/severe; } 4.4 \% \text { extremely } \\
\text { severe }\end{array}$ \\
\hline & & & & $\begin{array}{l}\text { Psychiatric morbidity (GHQ-12): } 64 \% \text { total; } 90.3 \% \text { HCW; } 49.1 \% \\
\text { non-HCW }\end{array}$ \\
\hline & & & & $\begin{array}{l}\text { PTSD/Stress (IES-R): at least moderate level on subscales: } 32.2 \% \\
\text { Intrusion; } 20.0 \% \text { avoidance; } 22.2 \% \text { hyperarousal }\end{array}$ \\
\hline Hong et al. (91) & China & 70 & $\begin{array}{l}\text { SARS-CoV-1/Post } \leq 1 y \text { and } \\
>1-4 y\end{array}$ & $\begin{array}{l}\text { PTSD/Stress (DSM-IV): } 44.1 \% \text { met criteria in at least one follow-up } \\
\text { visit }\end{array}$ \\
\hline \multirow[t]{4}{*}{ Lam et al. (92) } & Hong Kong & 181 (incl. HCW) & SARS-CoV-1/Post $>1-4 y$ & Depression (HADS/SCID): 35.6\%; 39\% \\
\hline & & & & $\begin{array}{l}\text { PTSD/Stress (IES-R): 27.9\% intrusion; 17.6\% avoidance; 33.5\% } \\
\text { hyperarousal }\end{array}$ \\
\hline & & & & $\begin{array}{l}\text { Further MHP (SCID): } 42.5 \% \text { at least one active psychiatric illness; } \\
54.5 \% \text { PTSD; } 36.4 \% \text { somatoform pain disorder; } 32.5 \% \text { panic disorder; } \\
\text { 15.6\% obsessive compulsive disorder }\end{array}$ \\
\hline & & & & Fatigue (CFQ/CFS): 40.3\%; $27.1 \%$ \\
\hline \multirow[t]{6}{*}{ Mak et al. (11) } & Hong Kong & 90 (incl. HCW) & SARS-CoV-1/Post >1-4y & $\begin{array}{l}\text { Since outbreak: } \\
\text { PTSD/Stress (IES-R/SCID): } 47.8 \%\end{array}$ \\
\hline & & & & $\begin{array}{l}\text { Further MHP (SCID): } 58.9 \% \text { any diagnosis; } 46.7 \% \text { depressive } \\
\text { disorder; } 21.1 \% \text { anxiety disorders }\end{array}$ \\
\hline & & & & $\begin{array}{l}30 \text { months post-SARS: } \\
\text { Anxiety (HADS): } 15.6 \% \text { moderate/severe anxiety }\end{array}$ \\
\hline & & & & Depression (HADS): 18.9\% moderate/severe depression \\
\hline & & & & PTSD/Stress (IES-R/SCID): 25.6\% \\
\hline & & & & $\begin{array}{l}\text { Further MHP (SCID): } 33.3 \% \text { any diagnosis; } 15.6 \% \text { depressive } \\
\text { disorder; } 14.6 \% \text { anxiety disorders }\end{array}$ \\
\hline Mak et al. (93) & Hong Kong & 90 (incl. HCW) & SARS-CoV-1/Post $>1-4 y$ & $\begin{array}{l}\text { PTSD (SCID): total of } 47.8 \% \text { at some time point after the SARS } \\
\text { outbreak; } 25.6 \% \text { at } 30 \text { months post-SARS }\end{array}$ \\
\hline
\end{tabular}

a PTSD, Post-traumatic stress disorder.

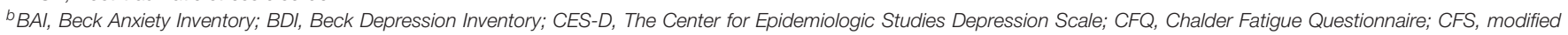

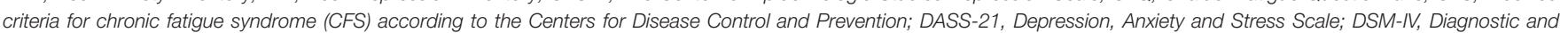

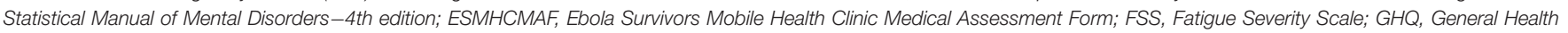

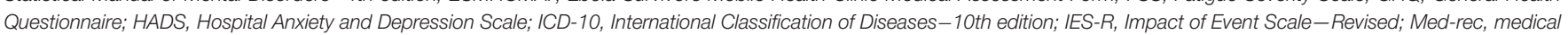

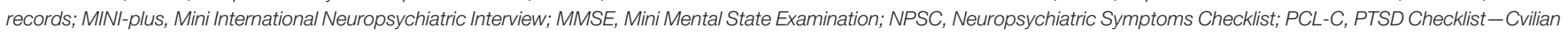
Version; PHQ-9, Patient Health Questionnaire; SCID, Structured Clinical Interview for DSM-IV; SCL-90-R, Symptom Checklist.

and trauma-related symptoms) as a host immunologic response to the infection (22). Mental health care interventions are expected to reduce symptoms such as PTSD (105). However, during epidemic scenarios care needs to be adapted to upcoming circumstances by respective governments in order to prevent or support individuals with MHP (106). In epidemic conditions, where consultation in-person is restricted there are important implications for digital health approaches. Online psychotherapy and consultation might help to improve access to mental health care, particularly in times of quarantine and isolation (107, 108). It does need to be highlighted that the effectiveness of online services for the improvement of mental health services requires further assessment (109). Consequently, the outbreak of SARS-CoV-2 calls for rapid reports and insights, as well as long-term health service research focusing on both remote and in-person mental health resources during epidemics $(110,111)$.

\section{Implications for HCW as a Highly Demanded Group}

Working conditions play an important role in mental health. For HCW, protective working conditions such as social support, constructive communication and staff training, and education have already been mentioned in some studies $(53,70$, 71). Employers should consider strengthening these resources by implementing support systems and coping management strategies. Besides such protective factors there might be even health promoting occupational aspects to be considered. For $\mathrm{HCW}$, the intent to help can buffer mental health-impairing consequences (74) but might be a rewarding factor in and of itself (112). It is also conceivable that enhanced public attention 
can trigger public appreciation of HCW. Furthermore, HCW could move to the political fore promoting improvements in the working conditions. Such rewarding aspects should be investigated in future studies.

\section{Implications for the General Public}

The importance of social support for mental health has been highlighted by several studies $(24,43,95)$. Digital communication with friends, relatives and colleagues might buffer the negative effects of loneliness and separation. Although most of the studies have highlighted stressors and protective factors to cope with these stressors, there might even be rewarding aspects in times of an epidemic. Some positive mental health-related factors like family support, mental health awareness and lifestyle changes such as time to rest, to relax or to exercise have already been investigated (43). During epidemics, a substantial proportion of individuals might be confronted with altered working conditions like teleworking, which is generally associated with pros and cons for mental health (113). Future studies should examine ways to reduce the negative impact of home-office situations in times of an epidemic crisis.

\section{Information Policies for Public Crisis Management}

Many studies have highlighted the role of timely and adequate information that should be provided (26). Epidemics with escalating case numbers and mass quarantine convey the impression of a serious personal threat and increase feelings of anxiety, loss of control and being trapped (114). The extensive mass and social media coverage is associated with public concerns and may contribute to negative psychological effects $(75,115)$. Appropriate information and education programs may not only help to decrease anxiety (45) but also benefit in adopting protective measures (116). Thus, adequate media is essential for the promotion of protective measures (115). Besides the responsibility of (health-) authorities to provide adequate information, it is necessary to understand the development of public attitudes to better target communication strategies, particularly with the rise of fake news and conspiracy theories (117). Furthermore, strengthening health literacy (118) appears to be important in enabling people to evaluate the relevant information. Consequently, it is of advantage to inform individuals that mild stress reactions may occur in such an epidemic that are not necessarily clinically relevant. However, a diagnostic clarification must be provided if justified by psychological strain. Generally, the application of health behavior theories in research of public attitudes and behaviors would enhance the development of public health interventions that address the mental health-impairing processes of an epidemic crisis.

\section{Addressing the Needs of Subpopulations in Public Health Policy}

With regard to the general public, the consideration of subpopulations was mainly neglected. For instance, people with mental illness (119) or children and families that might be victims of domestic violence, particularly in times of quarantine (120). Also, for the elderly, the effects of social distancing could lead to isolation, loneliness and severe mental health consequences (121). It is generally accepted to assume that people lacking resources (such as financial, cultural or social resources) might be more vulnerable within a crisis (122). Given this, future studies should examine mental-health effects for specific subpopulations. This would result in targeted interventions in these populations in addition to general public mental health approaches.

\section{Strengths and Limitations}

An important strength of our study is the inclusion of a broad range of populations that may be affected by MHP during or after an epidemic. This rapid review provides an essential overview of a highly relevant public health topic since the impact of impaired mental health itself on individuals, society and economy can be substantial. Furthermore, the data shown (Tables 1-3) allows for further interpretations and delivers insights to aspects that are of interest for researchers, practitioners and policy planning (e.g., country specific prevalence rates). Limitations may arise from the methods used to screen and extract the evidence for this article. To provide evidence in a timely manner, a rapid review is the method of choice as information need is immediate. This rapid review differs from a systematic review in several aspects. First, we focused our search strategy on PubMed and did not additionally screen reference lists of relevant articles. Second, the steps of screening and eligibility of research articles were performed by one author, respectively. Third, data was not extracted independently by two authors but were mutually controlled after extraction. Additionally, no quality assessment of the studies was conducted. Further limitations arise from the large heterogeneity and methodological issues (see section Mental Health Problems and Methodological Issues). At the same time, the heterogeneity of integrated studies is an asset, as they offer an extensive perspective on the studied issue.

\section{CONCLUSION}

In this rapid review of 74 original articles, we found a large range in prevalence rates of MHP such as anxiety, depression, post-traumatic stress symptoms or disorders, during and after epidemics across the general public, HCW, and survivors. MHP might be especially prominent among HCW and survivors that are directly affected by epidemics and face a real threat of infection and difficult circumstances like isolation/quarantine or difficult working conditions. As shown by various original studies, MHP across all populations can be substantially influenced by risk and protective factors, some of which are modifiable like social support and appropriate information by authorities. From a clinical point of view, policy makers and health care providers should be aware of potential short term or even persistent MHP. This is particularly relevant in planning of mental health infrastructure at large scale to encounter MHP elicited due to SARS-CoV-2 epidemic. Interventions should therefore rely on a comprehensive assessment combining risk factors for and symptoms of MHP considering their potential short or long term persistence. Short term MHP like stress reactions can generally be expected under the circumstances of an epidemic and should be distinguished from long term 
consequences or mental illness. Consequently, it may be required to develop and disseminate psychiatric programs based on the specific characteristics of the SARS-CoV-2 epidemic by integrating early diagnosis of determinants that anticipate a short or long-term course of treatment. During epidemics, mental health care needs to be adapted to changing circumstances in order to grant access and treatment to those in need. Digital mental health approaches can support access to care for the public. This allows for psychological monitoring and treatment when in-person consultations are not possible. Yet, digital health interventions are still in developmental stages and need further assessment. During lockdowns, they seem to be a relevant supplement to the provision of in-person mental health care. Furthermore, HCW that often account for a substantial fraction of virus cases need to be supported. However, health authorities and policy makers should keep in mind separating short-term acute stress reactions from long-term mental illness.

It is of note that many original studies used different approaches and show methodological diversity in the assessment of MHP, which at least partly explains the broad range of MHP. Thus, results should be treated with some caution since a comparison of prevalence rates across studies and assessment of magnitude of MHP is currently not possible. Future studies should monitor MHP with standardized methods and apply comparisons with country-specific norms and provide changes in prevalence rates in order to gain a better understanding of MHP, to learn about influential factors, and how to provide appropriate access to mental health care during epidemics. Although, this

\section{REFERENCES}

1. Peiris JS, Yuen KY, Osterhaus AD, Stohr K. The severe acute respiratory syndrome. N Engl J Med. (2003) 349:25. doi: 10.1056/NEJMra032498

2. Trifonov V, Khiabanian H, Rabadan R. Geographic dependence, surveillance, and origins of the 2009 influenza a (H1N1) virus. N Engl J Med. (2009) 361:2. doi: 10.1056/NEJMp0904572

3. Zaki AM, van Boheemen S, Bestebroer TM, Osterhaus AD, Fouchier RA. Isolation of a novel coronavirus from a man with pneumonia in Saudi Arabia. N Engl J Med. (2012) 367:19. doi: 10.1056/NEJMoa1211721

4. Gao R, Cao B, Hu Y, Feng Z, Wang D, Hu W, et al. Human infection with a novel avian-origin influenza a (H7N9) virus. $N$ Engl J Med. (2013) 368:20. doi: 10.1056/NEJMoa1304459

5. Baseler L, Chertow DS, Johnson KM, Feldmann H, Morens DM. The Pathogenesis of Ebola Virus Disease. Annu Rev Pathol. (2017) 12:387-418. doi: 10.1146/annurev-pathol-052016-100506

6. Wu JT, Leung K, Leung GM. Nowcasting and forecasting the potential domestic and international spread of the 2019-nCoV outbreak originating in Wuhan, China: a modelling study. Lancet. (2020) 395:10225. doi: 10.1016/s0140-6736(20)30260-9

7. Van Bortel T, Basnayake A, Wurie F, Jambai M, Koroma AS, Muana AT, et al. Psychosocial effects of an Ebola outbreak at individual, community and international levels. Bull World Health Organization. (2016) 94:3. doi: 10.2471/BLT.15.158543

8. Meo SA, Alhowikan AM, Al-Khlaiwi T, Meo IM, Halepoto DM, Iqbal $\mathrm{M}$, et al. Novel coronavirus 2019-nCoV: prevalence, biological and clinical characteristics comparison with SARS-CoV and MERS-CoV. Eur Rev Med Pharmacol Sci. (2020) 24:4. doi: 10.26355/eurrev_202002_20379

9. Wilder-Smith A, Freedman DO. Isolation, quarantine, social distancing and community containment: pivotal role for old-style public health measures was out of scope for this review, evidence of MHP in vulnerable populations such as children, the elderly especially when socially isolated or people with pre-existing mental illness seems to be scarce and should be covered in future studies.

\section{DATA AVAILABILITY STATEMENT}

The original contributions presented in the study are included in the article/supplementary materials, further inquiries can be directed to the corresponding author/s.

\section{AUTHOR CONTRIBUTIONS}

DR and SJZ contributed to the design of the study, data acquisition, data interpretation, manuscript development, and revisions. PK contributed to data acquisition, data interpretation, manuscript development and revisions. CA and $\mathrm{FKH}$ contributed to data acquisition and manuscript revisions. $\mathrm{CB}$ contributed to data interpretation and manuscript revision. AIL contributed to data interpretation, manuscript development and revisions. All authors approved the final version of the submitted manuscript.

\section{SUPPLEMENTARY MATERIAL}

The Supplementary Material for this article can be found online at: https://www.frontiersin.org/articles/10.3389/fpubh. 2020.560389/full\#supplementary-material

in the novel coronavirus (2019-nCoV) outbreak. J Travel Med. (2020) 27:2 doi: $10.1093 / \mathrm{jtm} / \mathrm{taaa} 020$

10. Smith RD. Responding to global infectious disease outbreaks: lessons from SARS on the role of risk perception, communication and management. Soc Sci Med. (2006) 63:12. doi: 10.1016/j.socscimed.2006.08.004

11. Mak IW, Chu CM, Pan PC, Yiu MG, Chan VL. Long-term psychiatric morbidities among SARS survivors. Gen Hosp Psychiatry. (2009) 31:4. doi: 10.1016/j.genhosppsych.2009.03.001

12. Dorn F, Fuest C, Göttert M, Krolage C, Lautenbacher S, Link S, et al. Die volkswirtschaftlichen Kosten des Corona-Shutdown für Deutschland: Eine Szenarienrechnung. ifo Schnelldienst. (2020) 4.

13. Rajkumar RP. COVID-19 and mental health: a review of the existing literature. Asian J Psychiatry. (2020) 52:102066. doi: 10.1016/j.ajp.2020.102066

14. Brooks SK, Webster RK, Smith LE, Woodland L, Wessely S, Greenberg $\mathrm{N}$, et al. The psychological impact of quarantine and how to reduce it: rapid review of the evidence. Lancet. (2020) 395:10227. doi: 10.1016/S0140-6736(20)30460-8

15. Chew QH, Wei KC, Vasoo S, Chua HC, Sim K. Narrative synthesis of psychological and coping responses towards emerging infectious disease outbreaks in the general population: practical considerations for the COVID-19 pandemic. Singapore Med J. (2020) 61:350-6. doi: 10.11622/smedj.2020046

16. Li S, Wang Y, Xue J, Zhao N, Zhu T. The impact of COVID-19 epidemic declaration on psychological consequences: a study on active weibo users. Int J Environ Res Public Health. (2020) 17:6. doi: 10.3390/ijerph170 62032

17. Fardin MA. COVID-19 and anxiety: a review of psychological impacts of infectious disease outbreaks. Arch Clin Infect Dis. (2020) 15:e102779. doi: 10.5812/archcid.102779 
18. Chan-Yeung M. Severe acute respiratory syndrome (SARS) and healthcare workers. Int J Occup Environ Health. (2004) 10:4. doi: 10.1179/oeh.2004.10.4.421

19. Suwantarat N, Apisarnthanarak A. Risks to healthcare workers with emerging diseases: lessons from MERS-CoV, Ebola, SARS, and avian flu. Curr Opinion Infect Dis. (2015) 28:4. doi: 10.1097/QCO.0000000000000183

20. Bai Y, Lin CC, Lin CY, Chen JY, Chue CM, Chou P. Survey of stress reactions among health care workers involved with the SARS outbreak. Psychiatr Serv. (2004) 55:9. doi: 10.1176/appi.ps.55.9.1055

21. Verma S, Mythily S, Chan YH, Deslypere JP, Teo EK, Chong SA. PostSARS psychological morbidity and stigma among general practitioners and traditional Chinese medicine practitioners in Singapore. Ann Acad Med Singapore. (2004) 33:6.

22. Troyer EA, Kohn JN, Hong S. Are we facing a crashing wave of neuropsychiatric sequelae of COVID-19? Neuropsychiatric symptoms and potential immunologic mechanisms. Brain Behav Immunity. (2020) 87:34-9. doi: 10.1016/j.bbi.2020.04.027

23. Tricco AC, Langlois EV, Straus SE, Alliance for Health P, Systems R, World Health O. Rapid Reviews to Strengthen Health Policy and Systems: A Practical Guide. Geneva: World Health Organization. (2017).

24. Cao W, Fang Z, Hou G, Han M, Xu X, Dong J, et al. The psychological impact of the COVID-19 epidemic on college students in China. Psychiatry Res. (2020) 287:112934. doi: 10.1016/j.psychres.2020.112934

25. Qiu J, Shen B, Zhao M, Wang Z, Xie B, Xu Y. A nationwide survey of psychological distress among Chinese people in the COVID-19 epidemic: implications and policy recommendations. General Psychiatry. (2020) 33:2. doi: 10.1136/gpsych-2020-100213

26. Wang C, Pan R, Wan X, Tan Y, Xu L, Ho CS, et al. Immediate psychological responses and associated factors during the initial stage of the 2019 coronavirus disease (COVID-19) epidemic among the general population in China. Int J Environ Res Public Health. (2020) 17:5. doi: 10.3390/ijerph17051729

27. Wang Y, Di Y, Ye J, Wei W. Study on the public psychological states and its related factors during the outbreak of coronavirus disease 2019 (COVID-19) in some regions of China. Psychol Health Med. (2020). doi: 10.1080/13548506.2020.1746817

28. Zhang Y, Ma ZF. Impact of the COVID-19 pandemic on mental health and quality of life among local residents in Liaoning Province, China: a cross-sectional study. Int J Environ Res Public Health. (2020) 17:7. doi: 10.3390/ijerph17072381

29. Kamara S, Walder A, Duncan J, Kabbedijk A, Hughes P, Muana A. Mental health care during the Ebola virus disease outbreak in Sierra Leone. Bull World Health Organization. (2017) 95:12. doi: 10.2471/BLT.16.190470

30. Koroma IB, Javadi D, Hann K, Harries AD, Smart F, Samba T. Non-communicable diseases in the Western Area District, Sierra Leone, following the Ebola outbreak. F1000Res. (2019) 8:795. doi: 10.12688/f1000research.18563.2

31. Betancourt TS, Brennan RT, Vinck P, VanderWeele TJ, Spencer-Walters D, Jeong J, et al. Associations between mental health and ebola-related health behaviors: a regionally representative cross-sectional survey in post-conflict Sierra Leone. PLoS Med. (2016) 13:8. doi: 10.1371/journal.pmed.1002073

32. Jalloh MF, Li W, Bunnell RE, Ethier KA, O'Leary A, Hageman KM, et al. Impact of Ebola experiences and risk perceptions on mental health in Sierra Leone, July 2015. BMJ Glob Health. (2018) 3:2. doi: 10.1136/bmjgh-2017-000471

33. Mollers M, Jonges M, Pas SD, van der Eijk AA, Dirksen K, Jansen C, et al. Follow-up of contacts of middle east respiratory syndrome coronavirusinfected returning travelers, the Netherlands, 2014. Emerg Infect Dis. (2015) 21:9. doi: 10.3201/eid2109.150560

34. Al-Rabiaah A, Temsah M-H, Al-Eyadhy AA, Hasan GM, Al-Zamil F, Al-Subaie S, et al. Middle East Respiratory Syndrome-Corona Virus (MERS-CoV) associated stress among medical students at a university teaching hospital in Saudi Arabia. J Infect Public Health. (2020) 13:5. doi: 10.1016/j.jiph.2020.01.005

35. Jeong H, Yim HW, Song YJ, Ki M, Min JA, Cho J, et al. Mental health status of people isolated due to middle east respiratory syndrome. Epidemiol Health. (2016) 38:e2016048. doi: 10.4178/epih.e20 16048
36. Rubin GJ, Amlôt R, Page L, Wessely S. Public perceptions, anxiety, and behaviour change in relation to the swine flu outbreak: cross sectional telephone survey. BMJ. (2009) 339:b2651. doi: 10.1136/bmj.b2651

37. Wang $Y, X u B, Z$ hao G, Cao R, He X, Fu S. Is quarantine related to immediate negative psychological consequences during the 2009 H1N1 epidemic? Gen Hosp Psychiatry. (2011) 33:1. doi: 10.1016/j.genhosppsych.2010.11.001

38. Xu J, Zheng Y, Wang M, Zhao J, Zhan Q, Fu M, et al. Predictors of symptoms of posttraumatic stress in Chinese university students during the 2009 H1N1 influenza pandemic. Med Sci Monit. (2011) 17:7. doi: 10.12659/msm. 881836

39. Leung GM, Lam TH, Ho LM, Ho SY, Chan BH, Wong IO, et al. The impact of community psychological responses on outbreak control for severe acute respiratory syndrome in Hong Kong. J Epidemiol Community Health. (2003) 57:11. doi: 10.1136/jech.57.11.857

40. Hawryluck L, Gold WL, Robinson S, Pogorski S, Galea S, Styra R. SARS control and psychological effects of quarantine, Toronto, Canada. Emerg Infect Dis. (2004) 10:7. doi: 10.3201/eid1007.030703

41. Quah SR, Hin-Peng L. Crisis prevention and management during SARS outbreak, Singapore. Emerg Infect Dis. (2004) 10:2. doi: 10.3201/eid1002.030418

42. Lau JTF, Yang X, Pang E, Tsui HY, Wong E, Wing YK. SARSrelated perceptions in Hong Kong. Emerg Infect Dis. (2005) 11:3. doi: 10.3201/eid1103.040675

43. Lau JT, Yang X, Tsui HY, Pang E, Wing YK. Positive mental healthrelated impacts of the SARS epidemic on the general public in Hong Kong and their associations with other negative impacts. J Infect. (2006) 53:2. doi: 10.1016/j.jinf.2005.10.019

44. Lee DT, Sahota D, Leung TN, Yip AS, Lee FF, Chung TK. Psychological responses of pregnant women to an infectious outbreak: a case-control study of the 2003 SARS outbreak in Hong Kong. J Psychosom Res. (2006) 61:5. doi: 10.1016/j.jpsychores.2006.08.005

45. Chan SS, So WK, Wong DC, Lee AC, Tiwari A. Improving older adults' knowledge and practice of preventive measures through a telephone health education during the SARS epidemic in Hong Kong: a pilot study. Int J Nurs Stud. (2007) 44:7. doi: 10.1016/j.ijnurstu.2006.04.019

46. Reynolds DL, Garay JR, Deamond SL, Moran MK, Gold W, Styra R. Understanding, compliance and psychological impact of the SARS quarantine experience. Epidemiol Infect. (2008) 136:7. doi: 10.1017/s0950268807009156

47. Sim K, Huak Chan Y, Chong PN, Chua HC, Wen Soon S. Psychosocial and coping responses within the community health care setting towards a national outbreak of an infectious disease. J Psychosom Res. (2010) 68:2. doi: 10.1016/j.jpsychores.2009.04.004

48. Lee TM, Chi I, Chung LW, Chou KL. Ageing and psychological response during the post-SARS period. Aging Ment Health. (2006) 10:3. doi: 10.1080/13607860600638545

49. Mihashi M, Otsubo Y, Yinjuan X, Nagatomi K, Hoshiko M, Ishitake T. Predictive factors of psychological disorder development during recovery following SARS outbreak. Health Psychol. (2009) 28:1. doi: 10.1037/a0013674

50. Peng EY, Lee MB, Tsai ST, Yang CC, Morisky DE, Tsai LT, et al. Population-based post-crisis psychological distress: an example from the SARS outbreak in Taiwan. J Formos Med Assoc. (2010) 109:7. doi: 10.1016/s0929-6646(10)60087-3

51. Lai J, Ma S, Wang Y, Cai Z, Hu J, Wei N, et al. Factors associated with mental health outcomes among health care workers exposed to coronavirus disease 2019. JAMA Network Open. (2020) 3:3. doi: 10.1001/jamanetworkopen.2020.3976

52. Sipos ML, Kim PY, Thomas SJ, Adler AB. U.S. service member deployment in response to the ebola crisis: the psychological perspective. Mil Med. (2018) 183:3-4. doi: 10.1093/milmed/usx042

53. Tang L, Pan L, Yuan L, Zha L. Prevalence and related factors of posttraumatic stress disorder among medical staff members exposed to H7N9 patients. Int J Nurs Sci. (2017) 4:1. doi: 10.1016/j.ijnss.2016.12.002

54. Lee SM, Kang WS, Cho AR, Kim T, Park JK. Psychological impact of the 2015 MERS outbreak on hospital workers and quarantined hemodialysis patients. Compr Psychiatry. (2018) 87:123-7. doi: 10.1016/j.comppsych.2018.10.003

55. Jung H, Jung SY, Lee MH, Kim MS. Assessing the presence of post-traumatic stress and turnover intention among nurses post-middle east respiratory 
syndrome outbreak: the importance of supervisor support. Workplace Health Saf. (2020) 68:337-45. doi: 10.1177/2165079919897693

56. Mishra P, Bhadauria US, Dasar PLNS, Kumar S, Lalani A, et al. Knowledge,attitude and anxiety towards pandemic flu a potential bio weapon among health professionals in Indore City. Przegl Epidemiol. (2016). 70:1.

57. Elizarraras-Rivas J, Vargas-Mendoza JE, Mayoral-Garcia M, MatadamasZarate C, Elizarraras-Cruz A, Taylor M, et al. Psychological response of family members of patients hospitalised for influenza A/H1N1 in Oaxaca, Mexico. BMC Psychiatry. (2010) 10:104. doi: 10.1186/1471-244x-10-104

58. Goulia P, Mantas C, Dimitroula D, Mantis D, Hyphantis T. General hospital staff worries, perceived sufficiency of information and associated psychological distress during the $\mathrm{A} / \mathrm{H} 1 \mathrm{~N} 1$ influenza pandemic. BMC Infect Dis. (2010) 10:322. doi: 10.1186/1471-2334-10-322

59. Chan AO, Huak CY. Psychological impact of the 2003 severe acute respiratory syndrome outbreak on health care workers in a medium size regional general hospital in Singapore. Occup Med (Lond). (2004) 54:3. doi: $10.1093 /$ occmed/kqh027

60. Chong MY, Wang WC, Hsieh WC, Lee CY, Chiu NM, Yeh WC, et al. Psychological impact of severe acute respiratory syndrome on health workers in a tertiary hospital. Br J Psychiatry. (2004) 185:127-33. doi: 10.1192/bjp.185.2.127

61. Nickell LA, Crighton EJ, Tracy CS, Al-Enazy H, Bolaji Y, Hanjrah S, et al. Psychosocial effects of SARS on hospital staff: survey of a large tertiary care institution. Cmaj. (2004) 170:5. doi: 10.1503/cmaj.1031077

62. Chen CS, Wu HY, Yang P, Yen CF. Psychological distress of nurses in Taiwan who worked during the outbreak of SARS. Psychiatr Serv. (2005) 56:1. doi: 10.1176/appi.ps.56.1.76

63. Lu YC, Shu BC, Chang YY, Lung FW. The mental health of hospital workers dealing with severe acute respiratory syndrome. Psychother Psychosom. (2006) 75:6. doi: 10.1159/000095443

64. Su TP, Lien TC, Yang CY, Su YL, Wang JH, Tsai SL, et al. Prevalence of psychiatric morbidity and psychological adaptation of the nurses in a structured SARS caring unit during outbreak: a prospective and periodic assessment study in Taiwan. J Psychiatr Res. (2007) 41:1-2. doi: 10.1016/j.jpsychires.2005.12.006

65. Tam CW, Pang EP, Lam LC, Chiu HF. Severe acute respiratory syndrome (SARS) in Hong Kong in 2003: stress and psychological impact among frontline healthcare workers. Psychol Med. (2004) 34:7. doi: 10.1017/s0033291704002247

66. Lung FW, Lu YC, Chang YY, Shu BC. Mental symptoms in different health professionals during the SARS attack: a follow-up study. Psychiatr Q. (2009) 80:2. doi: 10.1007/s11126-009-9095-5

67. Sim K, Chong PN, Chan YH, Soon WS. Severe acute respiratory syndromerelated psychiatric and posttraumatic morbidities and coping responses in medical staff within a primary health care setting in Singapore. J Clin Psychiatry. (2004) 65:8. doi: 10.4088/jcp.v65n0815

68. Phua DH, Tang HK, Tham KY. Coping responses of emergency physicians and nurses to the 2003 severe acute respiratory syndrome outbreak. Acad Emerg Med. (2005) 12:4. doi: 10.1197/j.aem.2004.11.015

69. Lin CY, Peng YC, Wu YH, Chang J, Chan CH, Yang DY. The psychological effect of severe acute respiratory syndrome on emergency department staff. Emerg Med J. (2007) 24:1. doi: 10.1136/emj.2006.035089

70. Lancee WJ, Maunder RG, Goldbloom DS. Prevalence of psychiatric disorders among Toronto hospital workers one to two years after the SARS outbreak. Psychiatr Serv. (2008) 59:1. doi: 10.1176/ps.2008.59.1.91

71. Maunder RG, Lancee WJ, Balderson KE, Bennett JP, Borgundvaag B, Evans $\mathrm{S}$, et al. Long-term psychological and occupational effects of providing hospital healthcare during SARS outbreak. Emerg Infect Dis. (2006) 12:12. doi: 10.3201/eid1212.060584

72. Wu P, Liu X, Fang Y, Fan B, Fuller CJ, Guan Z, et al. Alcohol abuse/dependence symptoms among hospital employees exposed to a SARS outbreak. Alcohol Alcohol. (2008) 43:6. doi: 10.1093/alcalc/ agn073

73. Wu P, Fang Y, Guan Z, Fan B, Kong J, Yao Z, et al. The psychological impact of the SARS epidemic on hospital employees in China: exposure, risk perception, and altruistic acceptance of risk. Can J Psychiatry. (2009) 54:5. doi: $10.1177 / 070674370905400504$
74. Liu X, Kakade M, Fuller CJ, Fan B, Fang Y, Kong J, et al. Depression after exposure to stressful events: lessons learned from the severe acute respiratory syndrome epidemic. Compr Psychiatry. (2012) 53:1. doi: 10.1016/j.comppsych.2011.02.003

75. Bo HX, Li W, Yang Y, Wang Y, Zhang Q, Cheung T, et al. Posttraumatic stress symptoms and attitude toward crisis mental health services among clinically stable patients with COVID-19 in China. Psychol Med. (2020) 1-2. doi: 10.1017/s0033291720000999. [Epub ahead of print].

76. Ji D, Ji YJ, Duan XZ, Li WG, Sun ZQ, Song XA, et al. Prevalence of psychological symptoms among Ebola survivors and healthcare workers during the 2014-2015 Ebola outbreak in Sierra Leone: a cross-sectional study. Oncotarget. (2017) 8:8. doi: 10.18632/oncotarget.14498

77. Howlett PJ, Walder AR, Lisk DR, Fitzgerald F, Sevalie S, Lado M, et al. Case series of severe neurologic sequelae of ebola virus disease during epidemic, Sierra Leone. Emerg Infect Dis. (2018) 24:8. doi: 10.3201/eid2408.171367

78. Etard JF, Sow MS, Leroy S, Toure A, Taverne B, Keita AK, et al. Multidisciplinary assessment of post-Ebola sequelae in Guinea (Postebogui): an observational cohort study. Lancet Infect Dis. (2017) 17:5. doi: 10.1016/s1473-3099(16)30516-3

79. Guetiya Wadoum RE, Samin A, Mafopa NG, Giovanetti M, Russo G, Turay $\mathrm{P}$, et al. Mobile health clinic for the medical management of clinical sequelae experienced by survivors of the 2013-2016 Ebola virus disease outbreak in Sierra Leone, West Africa. Eur J Clin Microbiol Infect Dis. (2017) 36:11. doi: 10.1007/s10096-017-3045-1

80. Keita MM, Taverne B, Sy Savane S, March L, Doukoure M, Sow MS, et al. Depressive symptoms among survivors of Ebola virus disease in Conakry (Guinea): preliminary results of the PostEboGui cohort. BMC Psychiatry. (2017) 17:1. doi: 10.1186/s12888-017-1280-8

81. Pers YM, Sow MS, Taverne B, March L, Izard S, Etard JF, et al. Characteristics of the musculoskeletal symptoms observed among survivors of Ebola virus disease in the Postebogui cohort in Guinea. Rheumatology. (2017) 56:12. doi: 10.1093/rheumatology/kex074

82. de St Maurice A, Ervin E, Orone R, Choi M, Dokubo EK, Rollin PE, et al. Care of ebola survivors and factors associated with clinical sequelae-monrovia, Liberia. Open Forum Infect Dis. (2018) 5:10. doi: 10.1093/ofid/ofy239

83. Kim HC, Yoo SY, Lee BH, Lee SH, Shin HS. Psychiatric findings in suspected and confirmed middle east respiratory syndrome patients quarantined in hospital: a retrospective chart analysis. Psychiatry Investig. (2018) 15:4. doi: $10.30773 /$ pi.2017.10.25.1

84. Lee SH, Shin HS, Park HY, Kim JL, Lee JJ, Lee H, et al. Depression as a mediator of chronic fatigue and post-traumatic stress symptoms in middle east respiratory syndrome survivors. Psychiatry Investig. (2019) 16:1. doi: $10.30773 /$ pi.2018.10.22.3

85. Cheng SK, Wong CW, Tsang J, Wong KC. Psychological distress and negative appraisals in survivors of severe acute respiratory syndrome (SARS). Psychol Med. (2004) 34:7. doi: 10.1017/s0033291704002272

86. Sheng B, Cheng SK, Lau KK, Li HL, Chan EL. The effects of disease severity, use of corticosteroids and social factors on neuropsychiatric complaints in severe acute respiratory syndrome (SARS) patients at acute and convalescent phases. Eur Psychiatry. (2005) 20:3. doi: 10.1016/j.eurpsy.2004.06.023

87. Wu KK, Chan SK, Ma TM. Posttraumatic stress after SARS. Emerg Infec Dis. (2005) 11:8. doi: 10.3201/eid1108.041083

88. Wu KK, Chan SK, Ma TM. Posttraumatic stress, anxiety, and depression in survivors of severe acute respiratory syndrome (SARS). J Trauma Stress. (2005) 18:1. doi: $10.1002 /$ jts.20004

89. Kwek SK, Chew WM, Ong KC, Ng AW, Lee LS, Kaw G, et al. Quality of life and psychological status in survivors of severe acute respiratory syndrome at 3 months postdischarge. J Psychosom Res. (2006) 60:5. doi: 10.1016/j.jpsychores.2005.08.020

90. Lee AM, Wong JG, McAlonan GM, Cheung V, Cheung C, Sham PC, et al. Stress and psychological distress among SARS survivors 1 year after the outbreak. Can J Psychiatry. (2007) 52:4. doi: 10.1177/070674370705200405

91. Hong X, Currier GW, Zhao X, Jiang Y, Zhou W, Wei J. Posttraumatic stress disorder in convalescent severe acute respiratory syndrome patients: a 4-year follow-up study. Gen Hosp Psychiatry. (2009) 31:6. doi: 10.1016/j.genhosppsych.2009.06.008

92. Lam MH, Wing YK, Yu MW, Leung CM, Ma RC, Kong AP, et al. Mental morbidities and chronic fatigue in severe acute respiratory 
syndrome survivors: long-term follow-up. Arch Intern Med. (2009) 169:22. doi: 10.1001/archinternmed.2009.384

93. Mak IW, Chu CM, Pan PC, Yiu MG, Ho SC, Chan VL. Risk factors for chronic post-traumatic stress disorder (PTSD) in SARS survivors. Gen Hosp Psychiatry. (2010) 32:6. doi: 10.1016/j.genhosppsych.2010.07.007

94. Sun L, Sun Z, Wu L, Zhu Z, Zhang F, Shang Z, et al. Prevalence and Risk Factors of Acute Posttraumatic Stress Symptoms during the COVID-19 Outbreak in Wuhan, China. medRxiv. (2020). doi: 10.1101/2020.03.06.20032425

95. Ko CH, Yen CF, Yen JY, Yang MJ. Psychosocial impact among the public of the severe acute respiratory syndrome epidemic in Taiwan. Psychiatry Clin Neurosci. (2006) 60:4. doi: 10.1111/j.1440-1819.2006.01522.x

96. Chua SE, Cheung V, McAlonan GM, Cheung C, Wong JW, Cheung EP, et al. Stress and psychological impact on SARS patients during the outbreak. Can J Psychiatry. (2004) 49:6. doi: 10.1177/070674370404900607

97. McAlonan GM, Lee AM, Cheung V, Cheung C, Tsang KW, Sham PC, et al. Immediate and sustained psychological impact of an emerging infectious disease outbreak on health care workers. Can J Psychiatry. (2007) 52:4. doi: 10.1177/070674370705200406

98. Maunder R, Hunter J, Vincent L, Bennett J, Peladeau N, Leszcz M, et al. The immediate psychological and occupational impact of the 2003 SARS outbreak in a teaching hospital. Cmaj. (2003) 168:10.

99. Cheng SK, Tsang JS, Ku KH, Wong CW, Ng YK. Psychiatric complications in patients with severe acute respiratory syndrome (SARS) during the acute treatment phase: a series of 10 cases. Br J Psychiatry. (2004) 184:359-60. doi: 10.1192/bjp.184.4.359

100. Pfefferbaum B, North CS. Mental Health and the Covid-19 Pandemic. N Engl J Med. (2020) 383:510-2. doi: 10.1056/NEJMp2008017

101. Carlier IV, Meuldijk D, Van Vliet IM, Van Fenema E, Van der Wee NJ, Zitman FG. Routine outcome monitoring and feedback on physical or mental health status: evidence and theory. J Eval Clin Pract. (2012) 18:1. doi: 10.1111/j.1365-2753.2010.01543.x

102. Rubanovich CK, Mohr DC, Schueller SM. Health app use among individuals with symptoms of depression and anxiety: a survey study with thematic coding. JMIR Ment Health. (2017) 4:2. doi: 10.2196/mental.7603

103. Lovejoy CA, Buch V, Maruthappu M. Technology and mental health: the role of artificial intelligence. Eur Psychiatry. (2019) 55:1-3. doi: 10.1016/j.eurpsy.2018.08.004

104. Wang J, Lloyd-Evans B, Giacco D, Forsyth R, Nebo C, Mann $\mathrm{F}$, et al. Social isolation in mental health: a conceptual and methodological review. Soc Psychiatry Psychiatr Epidemiol. (2017) 52:12. doi: 10.1007/s00127-017-1446-1

105. Torales J, O’Higgins M, Castaldelli-Maia JM, Ventriglio A. The outbreak of COVID-19 coronavirus and its impact on global mental health. Int J Soc Psychiatry. (2020) 66:4. doi: 10.1177/0020764020915212

106. Duan L, Zhu G. Psychological interventions for people affected by the COVID-19 epidemic. Lancet Psychiatry. (2020) 7:4. doi: 10.1016/s2215-0366(20)30073-0

107. Langarizadeh M, Tabatabaei MS, Tavakol K, Naghipour M, Rostami A, Moghbeli F. Telemental health care, an effective alternative to conventional mental care: a systematic review. Acta Inform Med. (2017) 25:4. doi: 10.5455/aim.2017.25.240-246

108. Tuerk PW, Keller SM, Acierno R. Treatment for anxiety and depression via clinical videoconferencing: evidence base and barriers to expanded access in practice. Focus (American Psychiatric Publishing). (2018) 16:4. doi: 10.1176/appi.focus.20180027

109. Kauer SD, Mangan C, Sanci L. Do online mental health services improve help-seeking for young people? A systematic review. J Med Internet Res. (2014) 16:3. doi: 10.2196/jmir.3103
110. Starace F, Ferrara M. COVID-19 disease emergency operational instructions for Mental Health Departments issued by the Italian Society of Epidemiological Psychiatry. Epidemiol Psychiatr Sci. (2020) 29:e116. doi: 10.1017/S2045796020000372

111. Wind TR, Rijkeboer M, Andersson G, Riper H. The COVID-19 pandemic: the 'black swan' for mental health care and a turning point for ehealth. Internet Interventions. (2020) 20:100317. doi: 10.1016/j.invent.2020. 100317

112. De Gieter S, De Cooman R, Pepermans R, Caers R, Du Bois C, Jegers M. Identifying nurses' rewards: a qualitative categorization study in Belgium. Hum Resour Health. (2006) 4:15. doi: 10.1186/1478-449 $1-4-15$

113. Mann S, Holdsworth L. The psychological impact of teleworking: stress, emotions and health. New Technol Work Employ. (2003) 18:3. doi: 10.1111/1468-005x.00121

114. Rubin GJ, Wessely S. The psychological effects of quarantining a city. Bmj. (2020) 368:m313. doi: 10.1136/bmj.m313

115. Rubin GJ, Potts HW, Michie S. The impact of communications about swine flu (influenza A H1N1v) on public responses to the outbreak: results from 36 national telephone surveys in the UK. Health Technol Assess. (2010) 14:34. doi: 10.3310/hta14340-03

116. Leung GM, Quah S, Ho LM, Ho SY, Hedley AJ, Lee HP, et al. Community psycho-behavioural surveillance and related impact on outbreak control in Hong Kong and Singapore during the SARS epidemic. Hong Kong Med J. (2009) 15(Suppl. 9):30-4.

117. Atlani-Duault L, Ward JK, Roy M, Morin C, Wilson A. Tracking online heroisation and blame in epidemics. Lancet Public Health. (2020) 5:3. doi: 10.1016/s2468-2667(20)30033-5

118. Kickbusch IS. Health literacy: addressing the health and education divide. Health Promot Int. (2001) 16:3. doi: 10.1093/heapro/16.3.289

119. Holmes EA, O'Connor RC, Perry VH, Tracey I, Wessely S, Arseneault L, et al. Multidisciplinary research priorities for the COVID-19 pandemic: a call for action for mental health science. Lancet Psychiatry. (2020) 7:547-60. doi: 10.1016/s2215-0366(20)30168-1

120. Campbell AM. An increasing risk of family violence during the Covid-19 pandemic: Strengthening community collaborations to save lives. Forensic Sci Int Reports. (2020). 2. doi: 10.1016/j.fsir.2020.100089

121. Newman MG, Zainal NH. The value of maintaining social connections for mental health in older people. Lancet Public Health. (2020) 5:1. doi: 10.1016/s2468-2667(19)30253-1

122. Hobfoll SE. The influence of culture, community, and the nested-self in the stress process: advancing conservation of resources theory. Appl Psychol. (2001) 50:3. doi: 10.1111/1464-0597.00062

Conflict of Interest: The reviewer AV declared a shared affiliation, with no collaboration, with several of the authors, PK and AIL, to the handling editor at the time of review.

The remaining authors declare that the research was conducted in the absence of any commercial or financial relationships that could be construed as a potential conflict of interest.

Copyright (C) 2020 Zürcher, Kerksieck, Adamus, Burr, Lehmann, Huber and Richter. This is an open-access article distributed under the terms of the Creative Commons Attribution License (CC BY). The use, distribution or reproduction in other forums is permitted, provided the original author(s) and the copyright owner(s) are credited and that the original publication in this journal is cited, in accordance with accepted academic practice. No use, distribution or reproduction is permitted which does not comply with these terms. 